\title{
Algorithm for the Sea Surface Wind Imaging Products of Fengyun-3C Meteorological Satellite MWRI
}

\author{
Dawei An, Fangli Dou, Peng Zhang \\ National Satellite Meteorological Center, China Meteorological Administration, Beijing, China \\ Email:andawei@cma.gov.cn
}

How to cite this paper: An, D.W., Dou, F.L. and Zhang, P. (2017) Algorithm for the Sea Surface Wind Imaging Products of Fengyun-3C Meteorological Satellite MWRI. Journal of Geoscience and Environment Protection, 5, 49-58. https://doi.org/10.4236/gep.2017.57006

Received: April 1, 2017

Accepted: July 10, 2017

Published: July 13, 2017

Copyright $\odot 2017$ by authors and Scientific Research Publishing Inc. This work is licensed under the Creative Commons Attribution International License (CC BY 4.0).

http://creativecommons.org/licenses/by/4.0/

c) $\underset{\mathrm{EY}}{(i)}$ Open Access

\begin{abstract}
This paper analyzes the sea surface backward thermal radiation data in the China Sea observed by the mmwave channel of FY3 MWRI, explains the reason for which the analysis method combined with multiple mmwave channels is conducive to wind inversion, uses the complex model of the two-scale randomly rough surface with foam scattering layer to calculate the backward heat emission, analyzes the different response characteristics of the thermal radiation characteristics of each channel with the change of the sea surface wind speed, and establishes the wind speed inversion model applying to the microwave radiometer, achieving better results than in previous studies. The sea surface medium-low wind speed precision standard deviation of new model reaches $1.2 \mathrm{~m} / \mathrm{s}(0-15 \mathrm{~m} / \mathrm{s})$; the inversion strong wind data are consistent with the island fixed buoys data, and the global sea surface wind speed image schematic diagram is given.
\end{abstract}

\section{Keywords}

Microwave Radiometer, Sea Surface Wind Speed, MWRI, Fengyun-3C Meteorological Satellite

\section{Introduction}

Throughout the world, many measuring methods for the sea surface wind field based on the satellite have been put into use, such as active radar and the common scatterometer (e.g. Seasat, ERS-I and II, ADEOS), and have achieved great success. However, the back scattering properties received by the scatterometer will tend to saturate, due to the influence of the sea surface foam under the high wind conditions, thus the highest operational sea wind speed [1] [2] [3] is only up to $25 \mathrm{~m} / \mathrm{s}$.

Wind field measurement using passive radiometer has been performed 
throughout the world. Goodberlet et al. developed the D-matrix algorithm, and carried out relevant research. Studies similar to mmwave imaging have also been performed in China. In Reference 12, Laurence N Connor and Paul S Chang adopted a TMI radiometer containing channel of $10.7 \mathrm{GHz}$ on the TRMM satellite, and achieved a good effect. However, due to the settings of the medium-low latitude orbit of TRMM satellite, it was difficult for the satellite to obtain the global sea surface wind speed, and its wide application is limited. Based on the research of Jin Yaqiu et al., it can be observed that the heat radiation effect received by the microwave radiometer [4] tends to saturate due to the disturbance from the foam scattering layer in strong wind areas. Therefore, the disturbance from the sea surface foam layer in the natural world is a major problem in wind inversion.

In this study, we used the MWRI of the Fengyun-3C meteorological satellite, which has channels of $10.65,18.7,23.8,36.5$ and $89 \mathrm{GHz}$, along with a large number of mmwave bands, including a high frequency channel similar to SSM/I, as well as a $10.7 \mathrm{GHz}$ channel which can pass through the atmosphere [5] [6] [7]. Furthermore, the Fengyun-3C meteorological satellite is a polar-orbiting operational satellite, thus it has the ability of obtaining the global sea surface wind speed. In this study, basing on the sea surface wind speed inversion model, we analyze the differences of backward heat radiation on the sea surface received by each channel with the change of the wind speed, set the channel configuration, establish the regression analysis method with new model coefficient, propose the wind reversion method, achieving a better result, and provide a schematic diagram for global sea surface wind speed. We also verify the accuracy and practicability of the model, providing a new concept for China to use a satellite-borne microwave radiometer to measure global sea surface wind speed [8] [9] [10].

\section{Modeling Research}

\subsection{Establishment and Verification of Medium-Low Wind Speed Model}

The basic assumption of the D-matrix arithmetic is that the required physical geography parameters, for example the sea surface wind speed (WS), can be represented as the linear combination of the measured brightness temperatures, i.e. the general type shown in Formula (1):

$$
W S=C_{0}+C_{1} T_{B 1}+C_{2} T_{B 2}+\cdots+C_{3} T_{B N}
$$

The variables in Formula (1) are the brightness temperatures, which are related to the selected channels. Coefficients of variables are determined by regression analysis. Based on previous research, this algorithm was corrected once again, and the new model which is fit to be used by MWRI for the sea surface wind speed inversion was established. This model applies to the range of $0-15$ $\mathrm{m} / \mathrm{s}$, i.e.:

$$
\begin{aligned}
W S= & C_{0}+C_{1} T_{B}(10.65 \mathrm{~V})+C_{2} T_{B}(10.65 \mathrm{H})+C_{3} T_{B}(18.7 \mathrm{~V}) \\
& +C_{4} T_{B}(23.8 \mathrm{~V})+C_{5} T_{B}(36.5 \mathrm{~V})+C_{6} T_{B}(36.5 \mathrm{H})
\end{aligned}
$$


It is well known that the global sea surface wind speed is uneven and unsymmetrical, and wind speeds of 95\% are mainly concentrated in the range of $2-15$ $\mathrm{m} / \mathrm{s}$ [11] [12]. Therefore, close attention should be given to the wind speed inversion.

We adopted the wind field inversion algorithm model shown in Formula (2). 1195, 1162 pieces of data from 2014 were collected through the fitting of the FY3B, C-MWRI and buoy, in order to generate the scatter plot for the MWRI wind speed shown in Figure 1, and the buoy wind speed of the corresponding time and space. The vertical coordinate represents the sea surface wind speed inversed by MWRI, the horizontal coordinate represents the buoy wind speed, the root-mean-square (RMS) of FY3B is $1.24 \mathrm{~m} / \mathrm{s}$, and the RMS of FY3C is 1.18 $\mathrm{m} / \mathrm{s}$.

\subsection{Research on Wind Speed Modeling}

By means of analyzing the phenomenon that backward heat radiation tends to saturate as a two-scale randomly rough surface of the sea surface foam layer, according to the different saturation characteristics in each channel, we extracted the wind sample data from recent years, and obtained the wind inversion algorithm using the regression and fitting method. The sea surface will be covered with one layer of crushed foam layer in the case of the strong wind, as shown in Figure 2, which will affect the heat radiation effect of the sea-surface roughness and the identification of wind.

Considering the atmosphere heat radiation, the radiation brightness temperature received by satellite MWRI should be as follows:

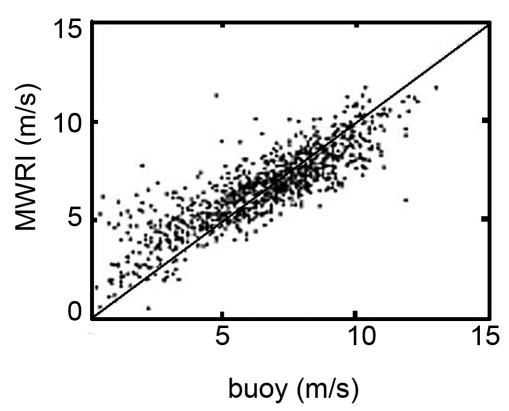

(a)

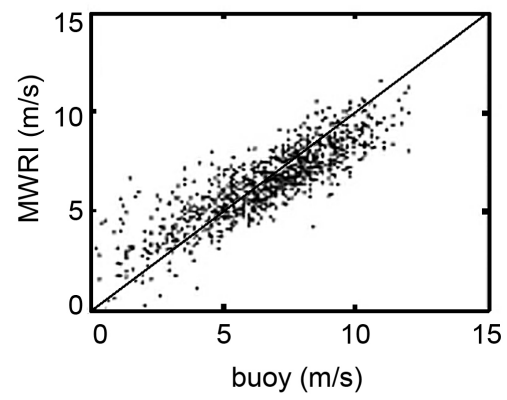

(b)

Figure 1. Scatterplot based on original model error. (a) FY3B; (b) FY3C.

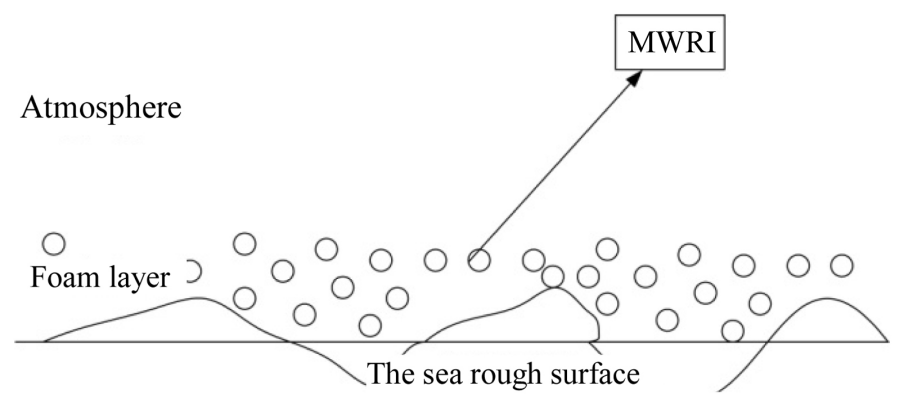

Figure 2. Rough sea surface model driven by strong wind. 


$$
T(\theta)=e(\theta) T_{s} e^{-\tau}+\left(1-e^{-\tau}\right)\left[1+r(\theta) e^{-\tau}\right] T_{a}
$$

where the vertical polarization heat emission rate is:

$$
e_{V}(\theta)=1-\frac{1}{4 \pi} \int_{0}^{2 \pi} \mathrm{d} \phi \int_{0}^{2 \pi} \mathrm{d} \theta^{\prime} \sin \theta^{\prime}\left[\gamma\left(\theta^{\prime}, \phi, \theta\right)\right],
$$

where the horizontal polarization heat emission rate is:

$$
e_{H}(\theta)=1-\frac{1}{4 \pi} \int_{0}^{2 \pi} \mathrm{d} \phi \int_{0}^{2 \pi} \mathrm{d} \theta^{\prime} \sin \theta^{\prime}\left[\gamma\left(\theta^{\prime}, \phi, \theta\right)\right],
$$

where the sea surface reflectivity $\gamma=1-e, \tau$ is the atmosphere opaqueness, $T_{s}$ is the sea surface physical temperature, and $T_{a}$ is the atmosphere physical temperature.

From Figure 3 it can be seen that the thermal radiations of the different frequency channels have different responses to the sea surface roughness (sea surface wind speed), and especially under the conditions of strong wind, the degree of each channel tending to saturate is different. If the single frequency measurement is used, then the back scattering properties and thermal radiation characteristics will tend to saturate, thus causing it to be difficult to carry out the wind inversion. If using multichannel combination fusion technology, we can improve the different characteristics of the thermal radiation for each channel responding to the sea surface wind speed tending to saturation, develop a combinational algorithm, and establish a new model suitable for the wind inversion.

The regression analysis is a statistical analysis method which is used to study the dependency relationship of one random variable $\mathrm{Y}$ with another $(\mathrm{X})$ or a set of variable $(\mathrm{X} 1, \mathrm{X} 2, \cdots, \mathrm{Xk})$. It requires a large number of data for analysis, must collect the global wind sample $(15-45 \mathrm{~m} / \mathrm{s})$ observed by the MWRI of FY-3C in orbit, and the coefficient can be adjusted slightly according to the channel frequency, sensitivity, etc. Finally, the new wind inversion model applying to MWRI is established.

The regression analysis model is as follows:

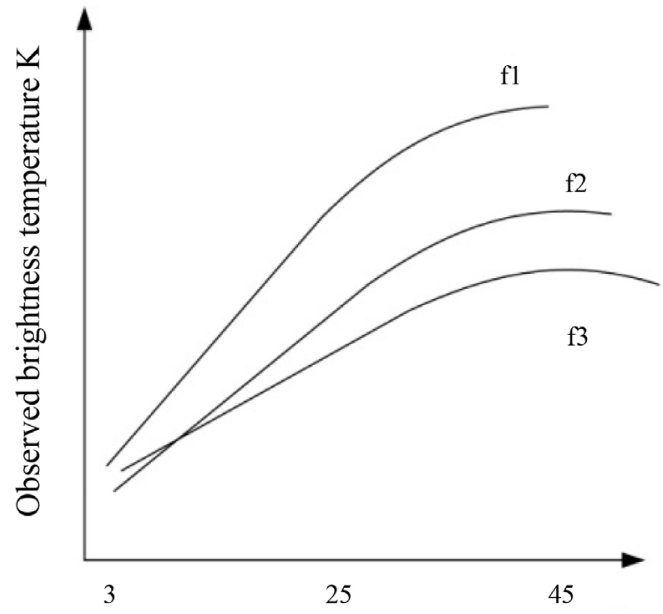

Figure 3. Response of thermal radiation received by each channel with the sea surface wind speed. 


$$
\begin{gathered}
\left\{\begin{array}{l}
W S=T C+C_{0} \\
E\left(C_{0}\right)=0, \operatorname{COV}\left(C_{0}, C_{0}\right)=\sigma^{2} I_{n}
\end{array}\right. \\
W S=\left[\begin{array}{c}
w s_{1} \\
w s_{2} \\
\vdots \\
w s_{n}
\end{array}\right], \quad T=\left[\begin{array}{ccccc}
1 & t_{11} & t_{12} & \cdots & t_{1 k} \\
1 & t_{21} & t_{22} & \cdots & t_{2 k} \\
\vdots & \vdots & \vdots & \cdots & \vdots \\
1 & t_{n 1} & t_{n 2} & \cdots & t_{n k}
\end{array}\right], \quad C=\left[\begin{array}{c}
c_{0} \\
c_{1} \\
\vdots \\
c_{k}
\end{array}\right], C_{0}=\left[\begin{array}{c}
c_{01} \\
c_{02} \\
\vdots \\
c_{0 n}
\end{array}\right],
\end{gathered}
$$

where $w s=C_{0}+C_{1} t_{1}+\cdots+C_{k} t_{k}$ is the regression plane equation, $T$ is the heat radiation brightness temperature, $C$ is the channel coefficient, and $W S$ is the wind speed. For the linear model $\left(W S, T C, \sigma^{2} I_{n}\right)$, the experimental value is mainly used to perform point estimation and hypothesis testing for the unknown parameters $C$ and $\sigma^{2}$ thereby the quantitative relation between ws and $t_{1} t_{2} \cdots t_{k}$ is established; $w s$ is predicted and controlled when $t_{1}=t_{01}, t_{2}=t_{02}$, $t_{k}=t_{0 k}$, namely interval estimation is performed for $w s$, and this process is known as least square thought. The quadratic sum of the deviation is $Q=\sum_{i=1}^{n}\left(w s_{i}-c \times t_{i}-c_{0}\right)$. On this basis, the linear regression equation coefficients of $c$ and $c_{0}$ are given, while the $c$ and $c_{0}$ values are within a certain range of the original model initial value:

$$
\left\{\begin{array}{l}
c=\frac{\sum_{i=1}^{n}\left(t_{i}-\bar{t}\right)\left(w s_{i}-\overline{w s}\right)}{\sum_{i=1}^{n}\left(t_{i}-\bar{t}\right)^{2}}=\frac{\sum_{i=1}^{n} t_{i} \times w s_{i}-n \bar{t} \times \overline{w s}}{\sum_{i=1}^{n} t_{i}^{2}-n \bar{t}^{2}} \\
c_{0}=\overline{w s}-c \times \bar{t} ; \cdots ; e_{i}<c_{i}<e e_{i} ; i \in[0, k]
\end{array}\right.
$$

Hereby, we must also consider the selection of the coefficient $c_{i}$ range, the responsive weight of the corresponding channel for the strong wind should minimize the $Q$ value (rather than making $Q$ value be 0 in the traditional regression method), and the floating range should be obtained using the weighted method. It can be seen that the frequency, sensitivity and calibration precisions of the channels corresponding to the instruments in Table 1 are different, and these differences will affect the brightness temperature data collected by the instrument. The weighted design of the differences is designed based on this difference, in order to perform the finite-field regression for the coefficient, namely the channel with least difference corresponds to the coefficient with smallest

Table 1. MWRI channel parameter indexes.

\begin{tabular}{ccccccc}
\hline \multirow{2}{*}{ Frequency (GHz) } & \multicolumn{2}{c}{10.65} & 18.7 & 23.8 & \multicolumn{2}{c}{36.5} \\
\cline { 2 - 7 } & $\mathrm{V}$ & $\mathrm{H}$ & $\mathrm{V}$ & $\mathrm{V}$ & $\mathrm{V}$ & $\mathrm{H}$ \\
\hline Sensitivity (K) & 0.5 & 0.54 & 0.5 & 0.8 & 0.31 & 0.5 \\
$\begin{array}{c}\text { Calibration } \\
\text { precision (K) }\end{array}$ & 1 & 1 & 1.5 & 2 & 2 & 2 \\
$\begin{array}{c}\text { Influence } \\
\text { coefficient }\end{array}$ & $c_{1}$ & $c_{2}$ & $c_{3}$ & $c_{5}$ & $c_{7}$ & $c_{8}$ \\
\hline
\end{tabular}


variation, and the channel with the greatest difference corresponds to the coefficient with the largest variation. The new model is obtained as follows:

$$
\begin{aligned}
W S= & C_{0}+C_{1} T_{B}(10 \mathrm{~V})+C_{2} T_{B}(10 \mathrm{H})+C_{3} T_{B}(19 \mathrm{~V})+C_{4} T_{B}(19 \mathrm{H})+C_{5} T_{B}(21 \mathrm{~V}) \\
& +C_{6} T_{B}(21 \mathrm{H})+C_{7} T_{B}\left((37 \mathrm{~V})+C_{8} T_{B}(37 \mathrm{H})+C_{9} T_{B}(89 \mathrm{~V})+C_{10} T_{B}(89 \mathrm{H})\right.
\end{aligned}
$$

The differences among channel frequency $f$, sensitivity $\Delta K$ and calibration precision $K$ are analyzed one by one. For the sensitivity $\Delta K$, the more sensitive the channel is, the higher the coefficient weight caused by the sensitivity will be; and for the calibration precision $K$, the higher the precision is, the higher the coefficient weight of this frequency $K$ point caused by the calibration precision will be. The specific coefficient is distributed by the weight of channel frequency $f$, sensitivity $\Delta K$ and calibration precision. In practical application, the $\mathrm{V}$ and $\mathrm{H}$ channels of $89 \mathrm{GHz}, \mathrm{H}$ channel of $18.7 \mathrm{GHz}$ and $\mathrm{H}$ channel of 23.8 $\mathrm{GHz}$ have smaller weight, which can be neglected, thus mainly the other effects of the channels should be analyzed.

For the monochromatic frequency $f$, the transmittance is the exponential function of the optical thickness:

$$
T_{\tilde{v}}\left(p_{s}, p\right)=\exp \left[-\frac{1}{g} \int_{p}^{p_{s}} k_{\tilde{v}}\left(p^{\prime}\right) q\left(p^{\prime}\right) \mathrm{d} p^{\prime}\right]
$$

From the two-dimensional function curvilinear relationship, it can be seen that near to the frequencies of 10,19 and $37 \mathrm{GHz}$, the transmittance shows the function characteristic of monotone decreasing, namely near this frequency point, the lower the frequency is, the smaller the attenuation will be; and the smaller the attenuation is, the smaller the disturbing influence from rainfall when the wind is measured will. In addition, the higher the frequency is, the greater the effect from rainfall will be; however, it is sensitive to the subtle variations of the sea surface roughness.

The horizontal coordinates in Figure 4 represent the brightness temperature error for each channel, in which the vertical coordinates represent the brightness temperature mean values, and the right Figure 4 represents the brightness temperature standard deviation. From the deviation mean, it can be seen that in the right Figure 4, the $\mathrm{H}$ channel of $10.65 \mathrm{GHz}, \mathrm{V}$ channel of $18.7 \mathrm{GHz}$ and $\mathrm{V}$ channel of $23.8 \mathrm{GHz}$ are not sensitive to the thermal radiation, while the $\mathrm{V}$
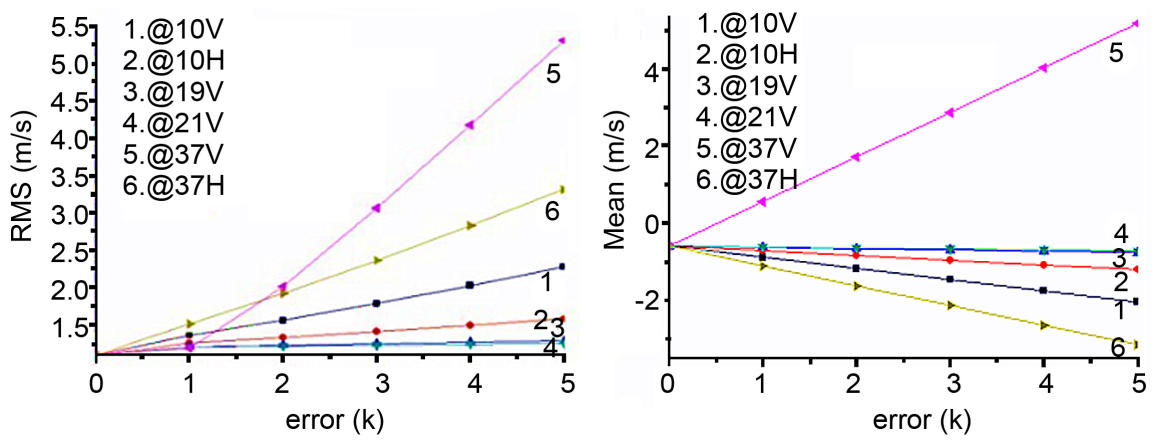

Figure 4. Comprehensive response effects of each channel for the thermal radiation; (left) mean, (right) standard deviation. 
channel of $10.65 \mathrm{GHz}$ and $\mathrm{H}$ and $\mathrm{V}$ channels of $36.5 \mathrm{GHz}$ are sensitive to the thermal radiation. From the standard deviation, it can be seen that in the left Figure 4, the $\mathrm{H}$ channel of $10.65 \mathrm{GHz}, \mathrm{V}$ channel of $18.7 \mathrm{GHz}$ and $\mathrm{V}$ channel of $23.8 \mathrm{GHz}$ are not sensitive to the thermal radiation, while the $\mathrm{V}$ channel of 10.65 $\mathrm{GHz}$ and $\mathrm{H}$ and $\mathrm{V}$ channels of $36.5 \mathrm{GHz}$ are sensitive to the thermal radiation. Additionally, the $\mathrm{V}$ channel of $36.5 \mathrm{GHz}$ is especially sensitive to the thermal radiation, and when the brightness temperature error of this channel reaches 5 $\mathrm{K}$, the deviation and standard deviation will both reach about $5 \mathrm{~K}$. The channel of $10.65 \mathrm{GHz}$ has the ability to pass through clouds and rain, which can greatly reflect the thermal radiation characteristics of the sea surface in cloudy and rainy areas.

\section{Inspection and Application Effect Evaluation}

The imaging products for global sea surface wind speed on July 7, 2014 are shown in the following Figure 5, which is obtained using the inversion of the medium-low wind speed model and high wind speed model, from which the low wind belt image in the north Pacific Ocean, the north Atlantic Ocean and the middle of the Indian Ocean can be seen clearly. The typhoon "NEOGURI" of the Western Pacific can be seen clearly, and in the small Figure 5 at bottom left, the internal profile of the NEOGURI typhoon can be seen clearly, including the typhoon eye area in the middle (the center wind power around eye area is higher than Level 14), spiral profile of the wind in periphery, seven levels of wind circle scale, and the image distribution of the super wind speed in the southeast area of the typhoon. This image product provides a strong guarantee for China's offshore wind monitoring and wind warning, with good practical application effects.

In Table 2, three super typhoons ("NEOGURI", "RAMMASUN" and "MATMO") in the Asian-Pacific region in 2014 are analyzed. The typhoons in the same region and same time, which are observed by the ASCAT scatterometer

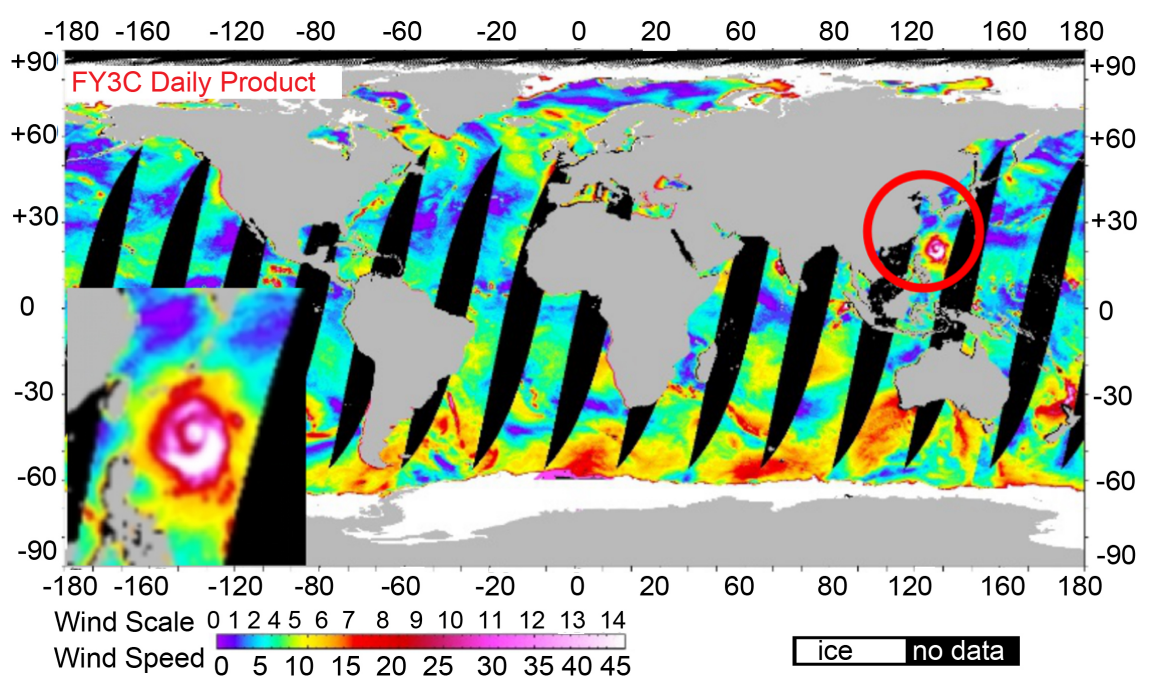

Figure 5. July 7, 2014 global sea surface wind speed distribution. 
carried by European METOP, are compared, while the wind speeds of the island fixed buoys in the same region and same time are used to compare and verify the maximum wind speed.

It can be seen that, due to the single frequency measurement of ASCAT, the storm foam layer under wind conditions causes the radar backscattering coefficient to be saturated, and all of the measured maximum wind speeds are $25 \mathrm{~m} / \mathrm{s}$. The maximum wind speeds inversed by FYS-WMRI are respectively 45, 45 and $43 \mathrm{~m} / \mathrm{s}$, and the actual maximum wind speeds measured by island fixed buoy are respectively 55, 52 and $40 \mathrm{~m} / \mathrm{s}$. It can be seen that the maximum wind speeds inversed by FY3-WMRI are closer to the actual values. This is crucial for coastal wind monitoring.

Typhoon Rammasun, shown in the middle of Figure 6, is distributed in the north area of the typhoon, which is coincident to the maximum wind speed area where the typhoon landed, as observed by the open sea island fixed buoy to the east of Haikou. From Typhoon Matmo, shown in the right of Figure 6, it can be seen that the maximum wind speed area is located in the north region, where it will affect Taidong County, Taiwan, and this analysis result is coincident to the maximum wind speed area where the typhoon actually landed.

\section{Conclusion}

This paper first introduced the development of the sea surface wind speed model inversed using a satellite-borne scatterometer and microwave radiometer and wind inversion problems throughout the world, as well as the preparation of the data source, including the introduction of the MWRI instrument and verification of the buoy. The brightness temperature data of Fengyun-3C meteorological

Table 2. 2014 super typhoon maximum wind speed.

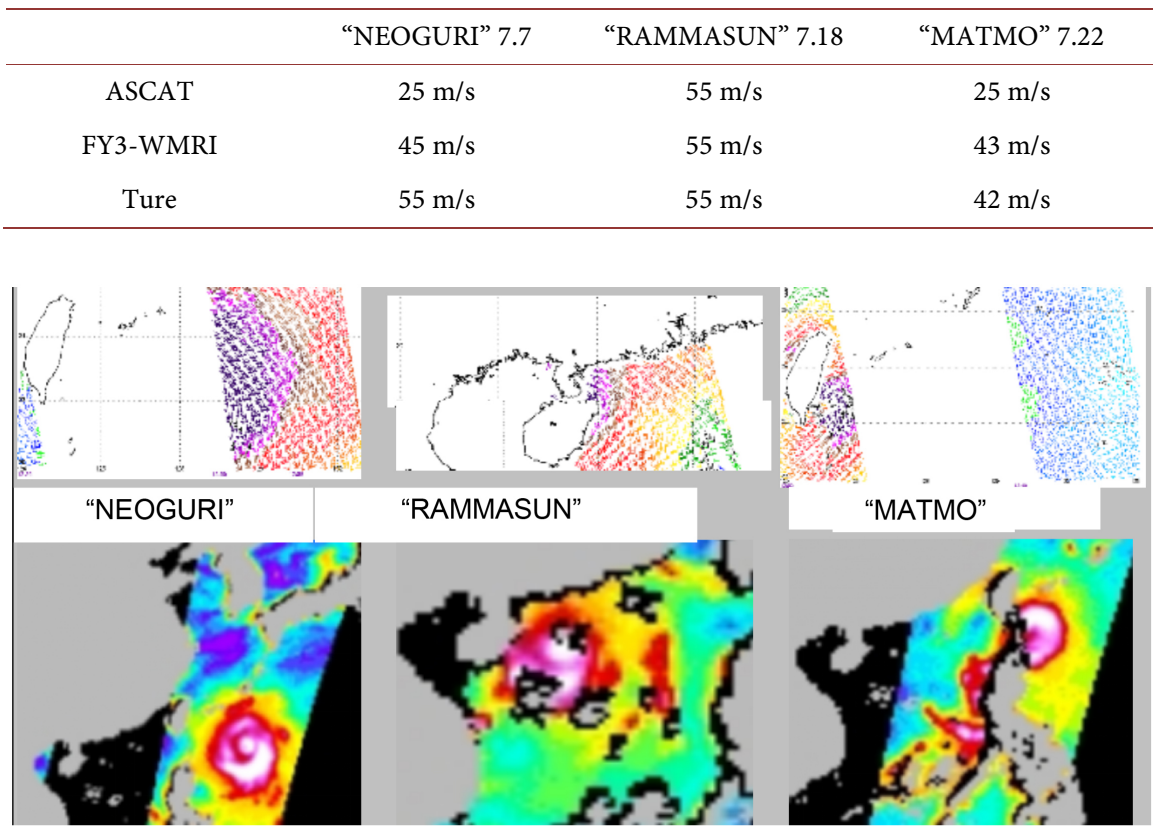

Figure 6. 2014 super typhoon inversion Winds (ASCAT and MWRI). 
satellite MWRI for one year were analyzed, providing the $0-15 \mathrm{~m} / \mathrm{s}$ wind speed inversion effect. This paper proved that the analysis method, combined with multiple channels, is helpful for the wind inversion. The backward heat emission was calculated, using the complex model of the two-scale randomly rough surface with foam scattering layer; the different response characteristics of the thermal radiation characteristics for each channel with the change of the sea surface wind speed were analyzed, and the wind speed inversion model applying to the microwave radiometer was established, achieving better results than in previous research. The sea surface medium-low wind speed precision standard deviation of the new model reaches $1.2 \mathrm{~m} / \mathrm{s}(0-15 \mathrm{~m} / \mathrm{s})$; the inversion strong wind data are consistent with the island fixed buoys data, and the global sea surface wind speed schematic diagram was given.

\section{References}

[1] Goodberlet, M.A. and Swift, C.T. (1992) Improved Retrievals from the DMSP Wind Speed Algorithm Under Adverse Weather Conditions. IEEE Transactions on Geoscience and Remote Sensing, 30, 1076-1077.

[2] Sun, L.E., Wang, J., Cui, T.W., Hao, Y.L. and Zhang, J. (2012) Statistical Inversion Algorithm for Sea Surface Temperature and Wind Speed of FY - 3B Microwave Imager. Journal of Remote Sensing, 6, 1262-1271.

[3] Chang, P.S. and Li, L. (1998) Ocean Surface Wind Speed and Direction Retrievals from the SSM/I. IEEE Transactions on Geoscience and Remote Sensing, 36, 1866-1871.

[4] Wang, R., Yan, W. and Shi, S.W. (2015) Sensitive Row Analysis of Environmental Parameters of Fully Polarized Microwave Radiometer. Journal of Remote Sensing, 3, 375-390.

[5] Wang, Z.Z. and Li, Y.L. (2009) Geophysical Parameters Retrieving Algorithm of AMSR. Journal of Remote Sensing, 29, 355-370.

[6] Zhang, G.F., Li, X.G. and Lou, G.W. (2007) Research on Passive Mmw Imaging Based on an Alternating Current Radiometer. Journal of Infrared and Millimeter Waves, 26, 461-464.

[7] Wang, F.Y., He, Y.J., Lu, Z.Z., Yuan, G.N. and Tang, Y.H. (2009) Algorithm Research on Inversion Ocean Surface Current Based on X Band Radar Image Sequence. Acta Geodaetica et Cartographica Sinica, 38, 443-449.

[8] Li, L.L., Guan, L. and Chen, R. (2006) Algorithm Research on Ocean Atmospheric Parameter Inversion Based on Satellite-Borne Microwave Radiometer. Periodical of Ocean University of China, 36, 205-211.

[9] Jin, Y.Q., Zhang, N.X., Huang, R.H. and Wang, Y. (1999) The Numerical Modeling of Correlation and Random Rough Surface Complex Model of the Sea Observed by Satellite-Borne E R S-1 and Multiple Channels SSM/I. Chinese Journal of Geophysics, 42, 452-459.

[10] Jin, Y.Q. (1998) Inversion of Satellite-Borne Microwave SSM/I for the Radiation Characteristic of South China Sea Surface Remote Sensing as well as Wind Field. Chinese Journal of Geophysics, 41, 30-37.

[11] An, D.W., Lu, F., Dou, F.L. and Zhang, P. (2015) Modeling and Quantitative Retrieval of Finite Field for The Tropical Sea Surface Wind Speed of The Fengyun-3 B Microwave Imager. Journal of Tropical Meteorology, 21, 84-91. 
[12] Connor, L.N. and Chang, P.S. (2000) Ocean Surface Wind Retrievals Using the TRMM Microwave Imager. IEEE Transactions on Geoscience and Remote Sensing, 38, No. 4.

Submit or recommend next manuscript to SCIRP and we will provide best service for you:

Accepting pre-submission inquiries through Email, Facebook, LinkedIn, Twitter, etc. A wide selection of journals (inclusive of 9 subjects, more than 200 journals) Providing 24-hour high-quality service User-friendly online submission system Fair and swift peer-review system Efficient typesetting and proofreading procedure Display of the result of downloads and visits, as well as the number of cited articles Maximum dissemination of your research work

Submit your manuscript at: http://papersubmission.scirp.org/ Or contact gep@scirp.org 